\title{
A systematic test of cognitive mapping, working-memory, and temporal discontiguity theories of hippocampal function
}

\author{
M. RASMUSSEN, C. A. BARNES, and B. L. McNAUGHTON \\ University of Colorado, Boulder, Colorado
}

\begin{abstract}
A completely between-subjects design was used to test three specific hypotheses of hippocam. pal function: O'Keefe and Nadel's cognitive mapping theory, Olton's working-memory theory, and Rawlins's temporal discontiguity hypothesis. The performance of rats with entorhinal lesions was compared with that of controls on a variety of spatial and nonspatial reference- and working-memory tasks using the same apparatus. The effects of massed versus distributed trials were also investigated. The entorhinal-lesioned animals were impaired on all tests of allocentric localization, regardless of the type of memory tested. The results were entirely consistent with the predictions of cognitive mapping theory, and failed to support the working-memory and temporal discontiguity theories. The results do not rule out the possibility that the cognitive mapping theory might, nevertheless, be a specific case of other, more general theories.
\end{abstract}

During the past decade, two major conflicting behavioral theories have predominated in the animal literature surrounding hippocampal function: the cognitive mapping (CM) theory of O'Keefe and Nadel $(1978,1979)$ and the working-memory (WM) theory (Honig, 1978) as articulated by Olton, Becker, and Handelmann (1979). In 1985, Rawlins introduced the temporal discontiguity (TD) hypothesis, which shares many of the predictions of WM theory but is in direct conflict with CM theory.

$\mathrm{CM}$ theory proposes that the hippocampus is the neural substrate for a memory system based on an allocentric spatial framework (or locale system). According to this theory, information about objects, places in the environment, and the relationships among objects and their locations is stored in this system. Thus, the cognitive map would permit an animal to respond flexibly to complex spatial problems. Egocentric response systems (or taxon systems) are ascribed to other parts of the brain. Thus, CM theory predicts that hippocampal damage would disrupt allocentric (place) tasks and would produce no impairment on nonspatial, or egocentric, tasks. WM theory proposes that the hippocampus is the substrate for a memory system that "emphasizes the temporal/personal context that separates one instance from another" (Olton, 1983 , p. 338). The WM component of a task is trialspecific. The temporal span of the memory may be brief or extended and the content of WM may be spatial or nonspatial. Reference memory (RM)

This work was included in a dissertation submitted in partial fulfillment of the requirements for the PhD degree (to M.R.) at the University of Colorado and was supported by PHS Grants AG-03376 (to C.A.B.) and NS-20331 (to B.L.M.). Address correspondence to M. Rasmussen, Neuropsychology Research Lab, Children's Hospital Research Center, 8001 Frost Street, San Diego, CA 92123. contains general information about the rules and procedures that is applicable to many different instances of the same class of events, and thus, does not require the current instance of the class to be distinguished from any other instance of the same class. (Olton, 1983, p. 337)

It is possible to design an experimental paradigm without a WM component; however, all behavioral tasks have RM components. According to WM theory, a pure RM task should not be affected by hippocampal damage. TD theory proposes that the hippocampus is a high-capacity, intermediate-term memory store (Marr, 1971) that processes stimuli from all modalities when the stimuli are to be associated with other events that occur separately in time. According to the original statement of this theory, when the temporal gap is small, a limited-capacity, short-term store located in another brain area should be able to support the necessary association. These two memory systems were proposed to operate in parallel. The predictions of Rawlins's (1985) hypothesis are similar to those of Olton et al.'s WM theory, except that for shortterm WM (i.e., with temporal gaps on the order of a few seconds), it should be possible for a limited-capacity, short-term memory buffer to support effective performance in the face of hippocampal damage.

In general, experiments designed to test these various hypotheses have been carried out using different experimental apparati, conditions, and subject pools (see Barnes, 1988). Not surprisingly, each theory has found a certain measure of experimental support. For example, fimbria-fornix-lesioned animals took five times as many trials to acquire a spatial version of a circular runway task as did controls, but were no different from controls on a nonspatial version of this task (O'Keefe, Nadel, Keightley, \& Kill, 1975). Furthermore, an enduring impairment was found in rats with total hippocampal lesions 
in the place version of the Morris water task (Morris, 1983; Morris, Garrud, Rawlins, \& O'Keefe, 1982), whereas lesioned rats performed as well as controls on a cued version of the task. On the other hand, acquisition and retention of spatial WM tasks are consistently impaired by hippocampal dysfunction (Jarrard, 1983; Jarrard, Okaichi, Steward, \& Goldschmidt, 1984; Morris et al. 1982; Olton et al., 1979). A number of studies have reported data indicative of a dissociation between RM and WM that does not fall along spatial/nonspatial lines after fimbria-fornix lesions (Olton, 1983; Olton \& Feustle, 1981; Olton \& Papas, 1979) or entorhinal lesions (Jarrard et al., 1984). To distinguish between the WM and TD theories, it is necessary to compare simple spatial and nonspatial alternation tasks in which the degree of temporal discontinuity is manipulated. A discrete-trial delayed-alternation task was adapted to this purpose by presenting versions with either short or long intervals between the information and choice phases and by imposing an experimenter-controlled activity during the delay (Rawlins \& Olton, 1982). The literature surrounding delayed alternation is ambiguous, however. Delays as short as $10 \mathrm{sec}$ in a T maze (Racine \& Kimble, 1965) or a barpress task (Niki, 1966) have revealed impairments following fimbria-fornix or hippocampal damage. On the other hand, only a transient deficit was found at the 10-sec interresponse interval on a $\mathrm{T}$ maze for rats with precommissural transection of the fornix (Thomas, 1978). It is far from clear, however, that this impairment necessarily resulted from a deficit in spanning temporal discontiguities. For instance, it is conceivable that an egocentric solution is possible if the animal is undisturbed, and that distraction or other movements during the interval forces an allocentric solution.

In the present experiments, we sought to evaluate the $\mathrm{CM}, \mathrm{WM}$, and TD theories using a completely betweensubjects design that utilized the same apparatus and in which every effort was made to treat the animals in a consistent manner throughout the study in order to minimize any additional intersubject variability introduced by between-subjects designs.

Entorhinal lesions were used in order to deafferent the hippocampus, producing a "hippocampal syndrome" without inducing the behavioral seizures that can accompany severe hippocampal damage. Previous studies have indicated that interruption of hippocampal input/output by either disruption of the fimbria-fornix or damage to the entorhinal cortex results in deficit patterns similar to those found after extensive damage to the hippocampus proper (Jarrard, 1980; Jarrard et al., 1984; Olton, Walker, \& Wolf, 1982).

\section{METHOD}

\section{Experimental Design}

The completely between-subjects lesion $\times$ task design was carried out using the same appetitive reinforcer, the same environment, and the same apparatus. The experiments were conducted in two phases. In Phase 1, nonspatial and spatial RM and WM were compared (refer to Table 1). Both distributed and massed trials were employed in different groups because memory consolidation and interference effects could not be determined in advance. Phase 2 consisted of the comparison of nonspatial (egocentric-taxon system) and spatial (allocentric--locale system) short-latency WM tasks that address the stated distinctions among all three theories. On the basis of the findings from Phase 1, only massed trials were ad-

Table 1

Experimental Design for Phase 1 Experiments

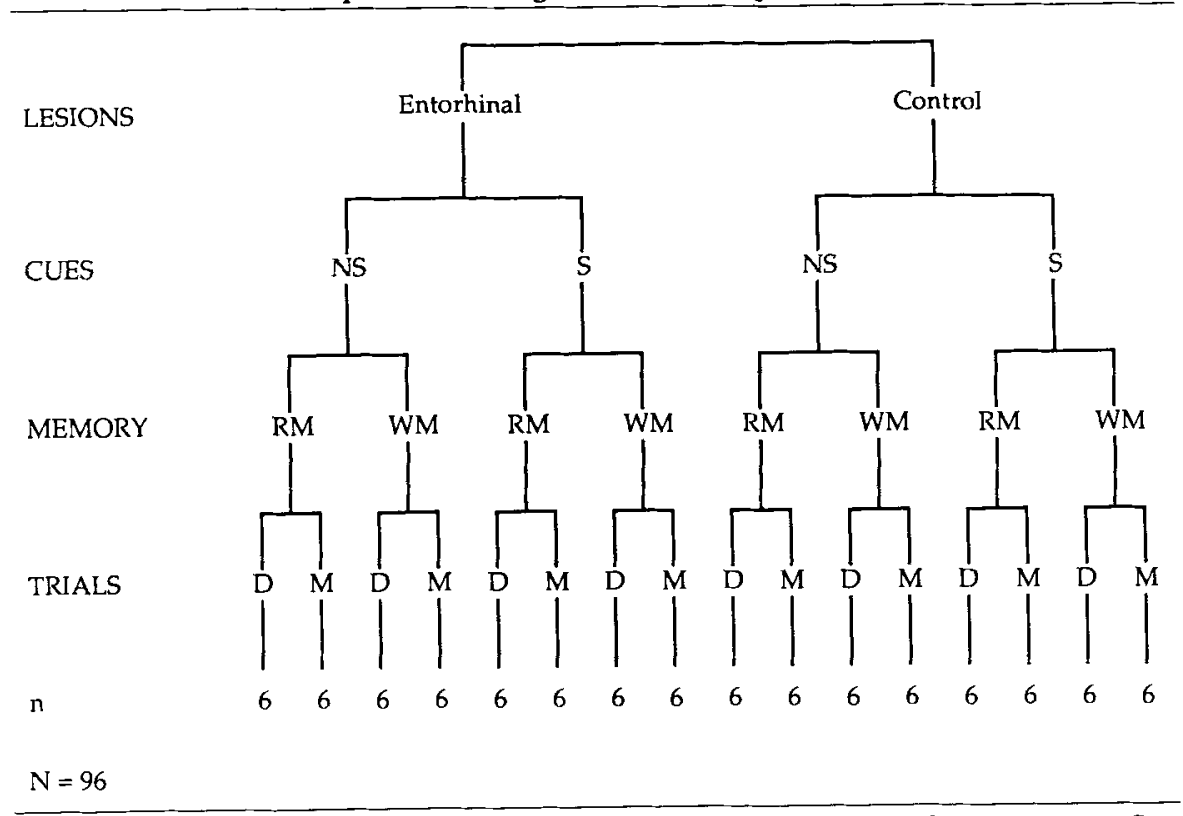

Note-- $\mathrm{D}=$ distributed trials, $\mathrm{M}=$ massed trials; $\mathrm{NS}=$ nonspatial, $\mathrm{RM}=$ reference memory, $\mathrm{S}=$ spatial, $\mathrm{WM}=$ working memory, $n=$ number of animals per cell, $N=$ total number of animals. 
ministered for the nonmatch-to-sample problems in Phase 2. Data are reported from a total of 24 animals in Phase 2.

\section{Animals}

One hundred twenty male Fischer-344 retired breeder rats (9-10 months of age at arrival) were obtained from Charles River Laboratories. They were housed in individual cages and maintained on Purina Rat Chow ad lib, supplemented by fresh fruit. All rats were kept in one of three adjacent colony rooms with the temperature held within the range of $21 \pm 3^{\circ} \mathrm{C}$ and a $12: 12 \mathrm{~h}$ light:dark cycle (lights on at 6:00 a.m.). All behavioral testing was carried out either within the dark cycle or within $1 \mathrm{~h}$ of the dark cycle.

Animals were excluded from the study for any of the following reasons: injury during shipping, poor health, failure to attain $90 \%$ of their original body weight following surgery, behavioral seizures, refusal of the reinforcer, or inadequate lesion as determined by preliminary histological analysis. It was necessary to exclude 6 animals (approximately 5\%) altogether. Replacement animals were evaluated in the subsequent behavioral testing sequence, along with additional control animals, to assure that no unnoticed extraneous variables biased the data. The animals were maintained at $80 \%-85 \%$ of their original body weights during testing.

\section{Surgery}

Food was removed $24 \mathrm{~h}$ prior to surgery and either water was removed or atropine injection was given to prevent anesthesia-related respiratory difficulties. The interval between surgery and behavioral testing was 14 days. During this time, the reinforcer (chocolate milk) was introduced to the animals in their home cages.

All surgery was performed under aseptic conditions and $33 \mathrm{mg} / \mathrm{kg}$ Nembutal was administered i.p. to achieve surgical anesthesia (supplemented as necessary with either Nembutal or sodium pentothal).

Electrolytic lesions of the entorhinal cortex were performed stereotaxically according to coordinates from Paxinos and Watson (1982). The lesion electrodes were constructed from $178-\mu \mathrm{m}$-diam stainless steel, teflon-coated wire, with approximately $400 \mu \mathrm{m}$ stripped from the tip. Five lesions were made in each hemisphere with the intention of destroying most of the entorhinal cortex. For the two anterior-most lesions, the electrode was lowered along a $10^{\circ}$ angle from vertical (parallel to the coronal plane): A-P: +1.5 (from lambda), M-L: $\pm 3.5 \mathrm{~mm}$ from the skull midline. The first lesion was made at $\mathrm{D}-\mathrm{V}-\mathbf{8 . 4}$, as measured from the preceding coordinates, by passing a $1.2 \mathrm{~mA}$ current for $12 \mathrm{sec}$. The second lesion was made along the same track at $\mathrm{D}-\mathrm{V}-7.4$ using a $1.0-\mathrm{mA}$ current for $10 \mathrm{sec}$. For the three posterior lesions, the electrode was lowered at an angle of $15^{\circ}$ from vertical, by passing a $1.5-\mathrm{mA}$ current for $15 \mathrm{sec}$ each. The $\mathrm{D}-\mathrm{V}$ coordinates were $-\mathbf{8 . 6},-7.5$, and -6.4 as measured from $A-P+1.0$, relative to lambda, and $\mathrm{M}-\mathrm{L} \pm 3.5 \mathrm{~mm}$ from the skull midline.

For sham control-lesioned animals, a scalp incision was made, and the wound was sutured. Following the surgery, $0.2 \mathrm{ml}$ of $\mathrm{Bi}$ cillin was injected intramuscularly $(0.1 \mathrm{ml}$ to each hind leg). The rats were maintained on a heating pad until they were ambulatory and able to ingest liquids or soft food spontaneously.

\section{Apparatus}

All behavioral testing was conducted on the same apparatus, a mechanized, elevated eight-arm radial maze. The working surface of the maze was constructed from black, matte-finished Plexiglas and was 30 in. above the baseplate. Each arm (23 in. long $x$ $2.25 \mathrm{in}$. wide) was supported separately by a post and there was a 9-in.-diam. stationary central platform. A 4-mm-high flange surrounded all flat surfaces of the maze. The arms were hinged, forming a 10 -in. bridge segment that could be lowered by remote control to create a gap so that the animals could be isolated on the central platform.
The configuration of the maze was altered according to the particular task, but the arrangement of the testing room remained the same throughout the entire study. There were abundant distal visual cues surrounding the maze and two sources of constant illumination provided diffuse, dim lighting in the room.

Visual-tactile cues, consisting of thin balsa strips covered with materials of various textures and visual characteristics, were constructed to fit the entire length of a single maze-arm. The cues included coarse-grained roofing paper painted with black and white longitudinal stripes, a smooth white background with gray rubber strips forming longitudinal stripes, and a soft fabric. The black maze surface itself served as the fourth cue.

\section{Procedure}

No specific maze-adasptation period was imposed. The rats were simply placed on the maze for their assigned task and permitted to explore. A structured system of prompting was employed if a rat failed to explore. However, data were not included in the anal$y$ sis until the animal reached a goal without prompting and the reinforcer was accepted from the foodwells (hereafter referred to as "consistent responding"). Because the difficulty level varied across tasks, the performance criterion was established empirically for each task, as a function of the learning curve. As the control group for a given task reached an asymptotic level, the performance of the least accurate control animal was used as the criterion for that task. The configuration of the maze for each task in Phase 1 is presented in Figure 1 and for Phase 2 in Figure 2. All distributed-trial conditions described below involved one trial per day.

Nonspatial RM. This task (Figure 1A) required selecting the one arm (of eight) on which a visual-tactile cue had been placed. Extramaze cues were irrelevant to task solution, because the goal arm was chosen at random for each trial. To begin a trial, the rat was placed in the center of the maze (as indicated by the arrow) with its initial orientation for each trial matched to the random sequence established for the spatial RM task on the basis of the angular separation between the initial position and the goal arm. The rat was allowed to choose among the arms until the goal arm had been selected. An error was recorded each time the rat entered a nongoal arm, and the rat was given a maximum of $5 \mathrm{~min}$ per trial. For the distributed-trial version (one trial per day), the criterion was set at no more than five errors in 5 consecutive days. For the massedtrial version [five trials per day, $10 \pm 5 \mathrm{~min}$ intertrial interval (ITI)], the criterion was no more than four errors in 3 consecutive days (15 consecutive trials).

Spatial RM. This task (Figure 1B) involved locating one arm (of eight) on the basis of extramaze cues. No arm inserts were employed. A particular goal arm was assigned to each rat for the entire course of testing. Other than this, the procedure was identical to that for nonspatial RM.

Nonspatial WM. This task (Figure 1C) required the rat to visit each of four visual-tactile cues once. The four arms selected for a given trial always formed a cross. However, either Arms 0, 2, 4, and 6 or Arms 1, 3, 5, and 7 were randomly selected. Extramaze cues were irrelevant to task solution. After each choice, the rat was removed and the cues were rearranged according to a predetermined, randomized pattern. The rat was returned to the central platform with its nose oriented halfway between a previously visited cue and an unvisited cue (as shown by the arrow). The interstimulus interval was $15 \mathrm{sec}$. An error was scored each time the rat returned to a previously visited cue. Half of the animals received distributed trials and half received massed trials (four trials per day, 15-min ITI). Testing continued until the following criteria had been met: for the distributed trials, no more than five errors in 5 consecutive days; for the massed trials, no more than four errors per day for 3 consecutive days, that is, no more than one error per trial, on average, for 12 consecutive trials (10-min time limit). 
A

\section{NONSPATIAL REFERENCE MEMORY}

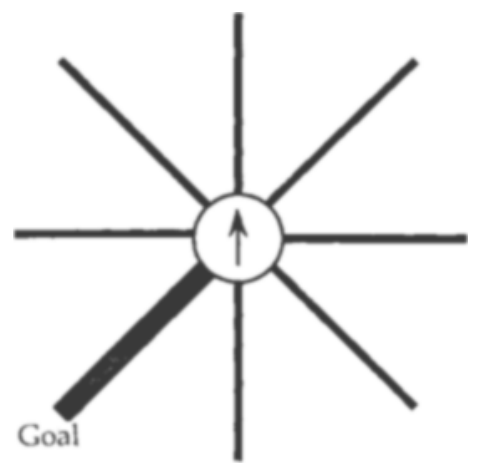

C

\section{NONSPATIAL WORKING MEMORY}

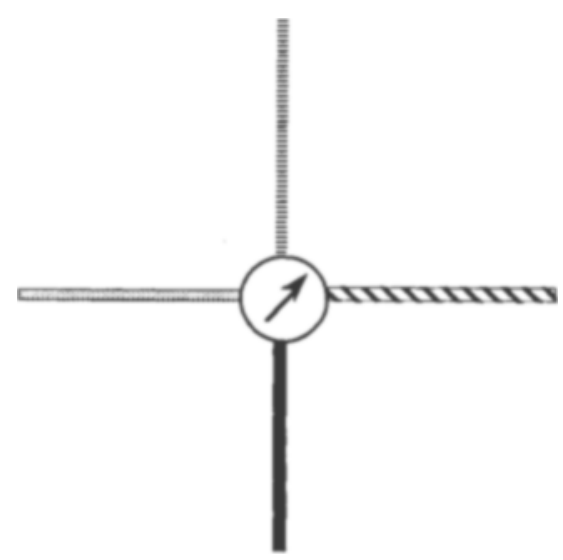

B

\section{SPATIAL REFERENCE MEMORY}

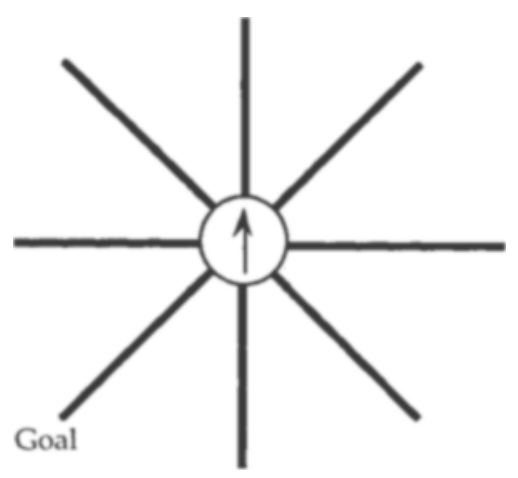

D

\section{SPATIAL WORKING MEMORY}

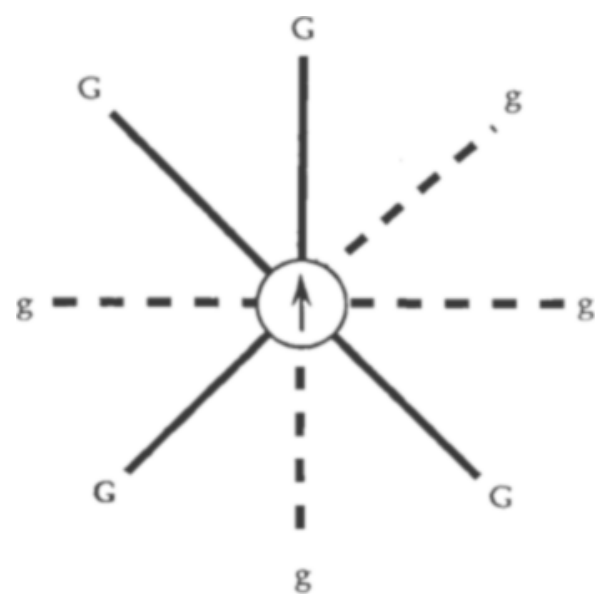

Figure 1. Maze configurations for Phase 1 experiments.

Spatial WM. This task (Figure 1D) required the rat to visit each of eight baited arms once, using distal extramaze cues. To begin a trial, the rat was placed on the center platform (as indicated by the arrow), in a randomly determined orientation, and exposed to four arms (solid lines marked G). After these arms had been visited, the remaining four arms (dashed lines marked $g$ ) were added and the rat was permitted to continue until all of the arms had been chosen or $5 \mathrm{~min}$ had elapsed. An error was scored each time the rat reentered any one of the eight arms. The following maze configurations were randomly presented in order to minimize the use of egocentric strategies: the pattern shown in Figure 1D, a "cross" pattern in which the arms were separated from one another by $90^{\circ}$, and an " $x$ " pattern in which two pairs of adjacent arms were separated by an angle of $135^{\circ}$.
Half of the animals received distributed trials and half received massed trials (five trials per day). The task time limit was 5 min per trial and for the massed trials, the ITI was $10 \pm 5 \mathrm{~min}$. For distributed trials, the criterion was no more than six errors in 4 consecutive days, and for massed trials the criterion was no more than five errors per day for 3 consecutive days, that is, no more than one error per trial, on average, for 15 consecutive trials.

Allocentric alternation. The allocentric task (Figure 2A) required the animal to alternate between places on the basis of extramaze cues. A "cross" configuration was used to prevent the rat from solving the task on the basis of turn direction. For the example shown, the east-west arms are the baited arms and the north-south arms are the start arms. However, for half of the trials, the start and goal arms were reversed. In Configuration $A$, the start arm 


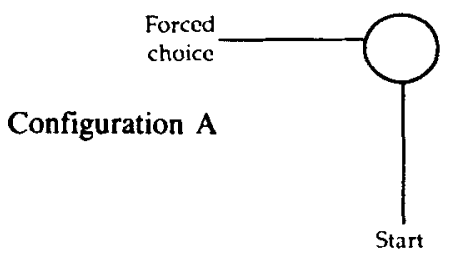

Step 1

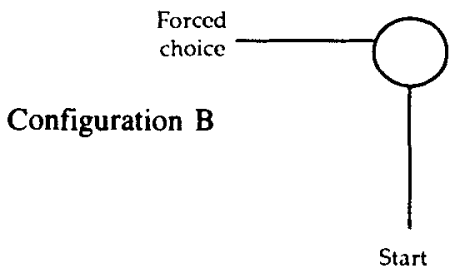

Step 1

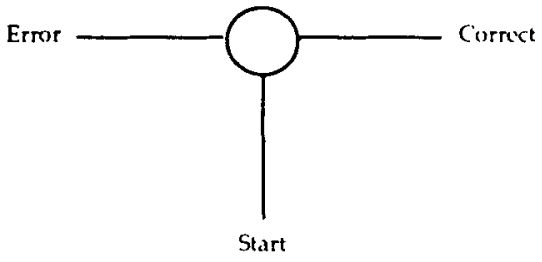

Step 2

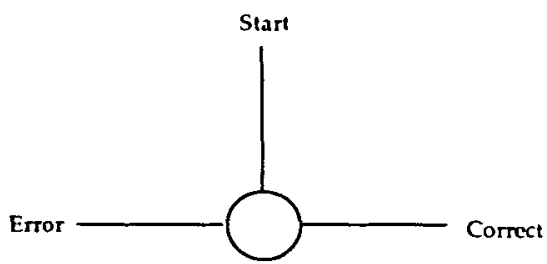

Step 2

B

EGOCENTRIC ALTERNATION

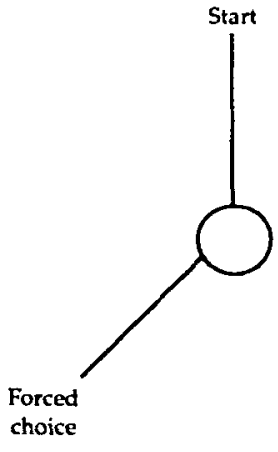

Step 1

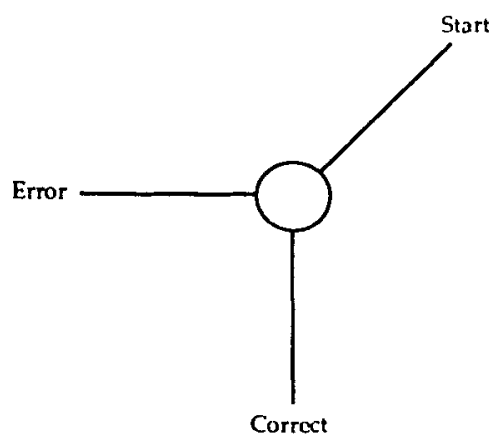

Step 2

Figure 2. Maze configurations for Phase 2 experiments.

was the same for Steps 1 and 2, whereas in Configuration B, the start arms were on the opposite side of the maze for the two steps.

To begin a trial, the rat was given access to one arm (the baited "forced-choice" arm) as shown in Step 1, and the rat was then removed from the maze before Step 2 was initiated. As quickly as possible, the rat was placed on the appropriate start arm and was permitted to choose between the two alternatives as shown in Step 2 (only the arm marked "correct"' in Figure 2A had the foodwell baited). An error was scored if the rat selected the previously forced arm. The rat was then replaced on the start arm for Step 2, following a 6-10 sec delay, and was allowed to choose again until the correct choice had been made. Thus, it was possible for an animal to make several errors on a given trial. During the delay, the animals were held by the experimenter. The criterion for advancement to the next delay condition was set at no more than five errors in 3 consecutive days ( 15 consecutive trials). Entorhinal-lesioned rats that failed to meet this criterion in 10 days were moved to the next delay condition. Data were collected for a maximum of 5 days for each subsequent delay condition.

Egocentric alternation. This task required the animal to alternate between goals on the basis of turn direction. The Y-maze configuration shown in Figure 2B was used. In this example, the rat had to turn left for the forced choice (Step 1). In Step 2, the starting arm was changed and two choices were provided. The arm that the rat had visited in Step 1 (which was no longer available) was located halfway between the two choices offered in Step 2. To make the correct choice in this example, the rat was required to turn right. There were eight possible starting arms, selected randomly across trials. The direction of the forced choice in Step 1 was random for a given trial. The rest of the procedure was the same as for allocentric 
alternation, except that the animals were returned to their cages during the 30- and 60-sec delays. The criterion for advancement to the next delay condition was set at no more than six errors in 3 consecutive days ( 15 consecutive trials). A maximum of 15 days was allowed for the zero-delay condition. Data were collected for a maximum of 6 days for each subsequent delay condition.

\section{Histology}

The rats were anesthetized with Nembutal and perfused transcardially with a $10 \%$ Formalin solution in physiological saline. The brains were blocked, embedded in either egg yoke or gelatin, and stored in a $30 \%$ sucrose-Formalin solution. A one in four series was sectioned in the coronal plane at $40 \mu \mathrm{m}$ throughout the damaged area (including $500 \mu \mathrm{m}$ before and after any observable damage) and was stained with cresyl violet.

Prior to data analysis, the six best lesions were chosen for each of the groups. The minimal acceptable lesion to the entorhinal cortex was $80 \%$ destruction of the medial entorhinal cortex including the cell area in which the medial perforant path originates. No lesion with damage to the hippocampus proper extending beyond the most posterior area was accepted.

\section{Behavioral Data Analysis}

To establish the degree to which the animals were able to master each task, the percent correct during the final set of trials was calculated. Two measures of performance (the number of days to criterion and the number of errors to criterion) were calculated and the group means for each measure were compared using $t$ tests (alpha $=.050$ ) for each task separately. The results of the latter measure were then displayed in histograms for each task.

\section{RESULTS}

\section{Controls}

The mean number of errors to criterion was compared for operated and unoperated controls for each behavioral task, using $t$ tests. Since there were no significant differences between any of the groups, the control data were pooled.

\section{Histology}

Figure 3 shows the range of entorhinal lesions used in the study. The largest lesions destroyed almost the entire extent of the medial entorhinal cortex and about $30 \%$ of the lateral entorhinal cortex. In addition, approximately $50 \%$ of the parasubiculum, $10 \%$ of the presubiculum, and a considerable amount of subiculum and fascia dentata in the ventroposterior region were destroyed. An intermediate group of lesions destroyed between $80 \%$ and $90 \%$ of the medial and $10 \%-25 \%$ of the lateral entorhinal cortex, approximately $20 \%-40 \%$ of the parasubiculum, $10 \%-15 \%$ of the subiculum, and no more than $10 \%$ of the fascia dentata. The smallest lesions left approximately $0.5-1.0-\mathrm{mm}$ bands of medial entorhinal cortex in the most ventral portion and barely encroached upon the lateral entorhinal cortex. Far less parasubiculum, presubiculum, and subiculum were destroyed, and the fascia dentata was intact. The brains were ranked according to the amount of damage. Approximately $37 \%$ had bilateral lesions within the large category, $22 \%$ were judged to be large on one side and intermediate on the other side, $21 \%$ were intermediate bilaterally, $16 \%$ were intermediate on one

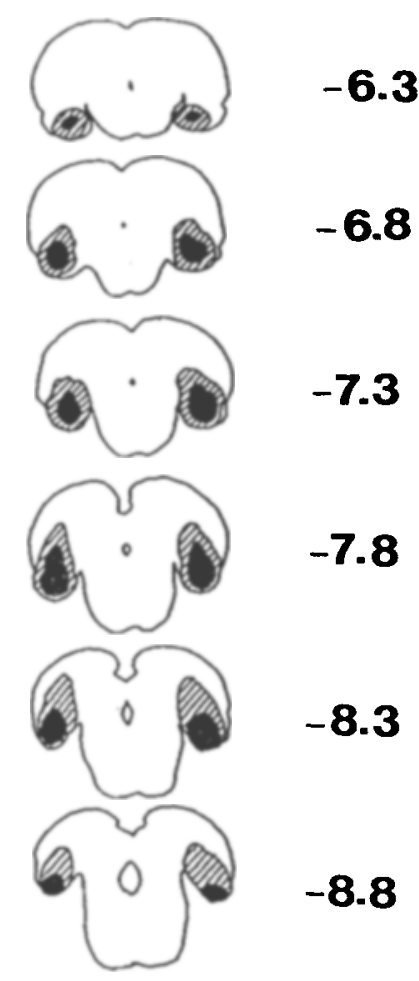

Figure 3. Schematic diagram of the extent of entorhinal lesions. The cross-hatched area represents the largest lesion, and the black area represents the smallest lesion. The numerals indicate the A-P coordinate of each section as measured from Bregma in millimeters.

side and small on the other side, and 5\% were judged to be small bilaterally.

The positive correlation (.231) for the comparison between lesion size and the severity of impairment failed to reach significance, nor was a relationship found between the amount of incidental damage to Area 17 and severity of the impairment $(r=-0.081)$. The latter findings indicate that very little of the variance could be accounted for by damage to visual cortex.

\section{Behavioral Testing}

Nonspatial RM. For the distributed-trials groups, control and entorhinal-lesioned rats were able to meet the criterion within 8 days of consistent performance. Controls took significantly more days to reach criterion than did the entorhinal group $[t(10)=2.272, p=.046]$. During the last 5 days, controls achieved a response accuracy of $93 \%$, and the entorhinal group, $92 \%$.

Figure 4a shows the mean number of errors to criterion made by each group. These means were derived from the sums of all scorable errors made by each animal. The two groups did not differ in the mean number of errors to criterion $[t(10)=0.725, p=.485]$.

For the massed-trials groups, controls and entorhinallesioned rats were able to meet the criterion within a short time (within 5 days of consistent performance). The two groups did not differ in either the mean number of days 
to criterion $[t(10)=0.739, p=.477]$ or the mean number of errors $[t(10)=1.276, p=.231]$, as shown in Figure $4 \mathrm{~b}$. During the last 3 days, both groups achieved a response accuracy of $98 \%$.

In summary, there were no significant differences between the entorhinal and control groups for any of the nonspatial RM measures, except that controls took more days than entorhinal-lesioned rats to reach criterion on the distributed-trial version.

Spatial RM. For the distributed-trials groups, all controls were able to meet the criterion. Of the entorhinal rats, only 4 of 6 acquired the task by Day 22. The range for the total acquisition period was from 7 to 20 days for the animals that were able to meet criterion. The entorhinal group took significantly more days to reach criterion than did controls $[t(10)=3.051, p=.012]$. Furthermore, during the final 5 days, controls made $95 \%$ and entorhinal rats made $78 \%$ correct responses. Figure 5 a shows the mean number of errors made by each group of animals. The lesioned rats made significantly more errors than did the controls $[t(10)=2.854, p=.017]$.

For the massed-trials groups, all controls were able to meet the criterion. Of the entorhinal rats, only 4 of 6 acquired the task by Day 15 ( 75 trials). The range was from 4 to 15 days (20-75 trials). The entorhinal group took significantly longer to reach criterion than did the controls $[t(10)=6.579, p<.001]$. However, during the final 3 days, controls were correct $98 \%$ and entorhinal rats $93 \%$ of the time. Figure $5 \mathrm{~b}$ shows that the entorhinal group made significantly more errors than did the controls $[t(10)=5.913, p<.001]$.

In summary, entorhinal lesions produced impairment in both the number of days and the number of errors to criterion. Perseverative responding was common among the lesioned animals during the early trials, especially for the massed trials. This behavior continued throughout testing for a majority of the lesioned animals. The overall impairment, as measured by the number of errors to criterion, was much more pronounced on the massed-trial version. However, the accuracy level was better for the massed-trial group by the end of training.

Nonspatial WM. For the distributed-trials groups, both control and entorhinal-lesioned rats met the criterion. The range to criterion was 5-27 days. Although entorhinal rats took 12.7 days to reach criterion, on average, and controls took only 8.8 days, the difference in the means failed to reach significance $[t(10)=1.669, p=.126]$. During the last 5 days, controls and entorhinals achieved only $79 \%$ and $76 \%$ accuracy, respectively. Figure $6 a$ shows the mean number of errors to criterion. The difference in these means was not technically significant $[t(10)=$ 2.214, $p=.051]$.

For the massed-trials groups, both control and entorhinal-lesioned rats were able to meet the criterion. The range to criterion was 6-23 days. There was no difference between the groups in the mean number of days to criterion $[t(10)=0.012, p=.991]$. During the final 3 days, controls made $83 \%$ and entorhinal-lesioned rats made $85 \%$ correct choices. Figure $6 \mathrm{~b}$ shows that the entorhinal group made no more errors than did the control group $[t(10)=0.825, p=.428]$.

In summary, there was a borderline-significant trend in the direction of more errors for entorhinal rats on the distributed-trial version (relative to controls), largely due to early perseverative responding of the lesioned animals. Early perseverative responding also occurred during the massed-trial version but did not have a great impact on overall error measures because of the larger number of trials. The entorhinal group was clearly unimpaired on all measures of performance for the massed-trial version.

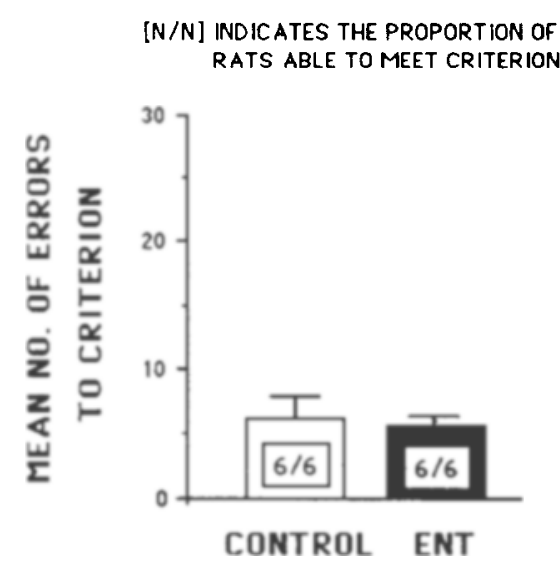

a. DISTRIBUTED TRIALS

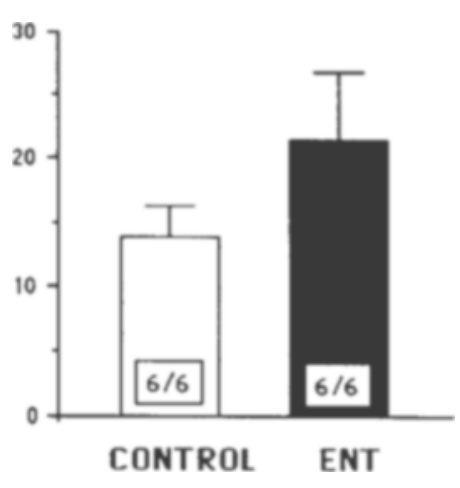

b. MASSED TRIALS

\section{EXPERIMENTAL GROUP}

Figure 4. Mean number of errors to criterion in the nonspatial reference-memory task. Bars represent the standard error of the mean. ENT = entorhinal-lesioned rats. 


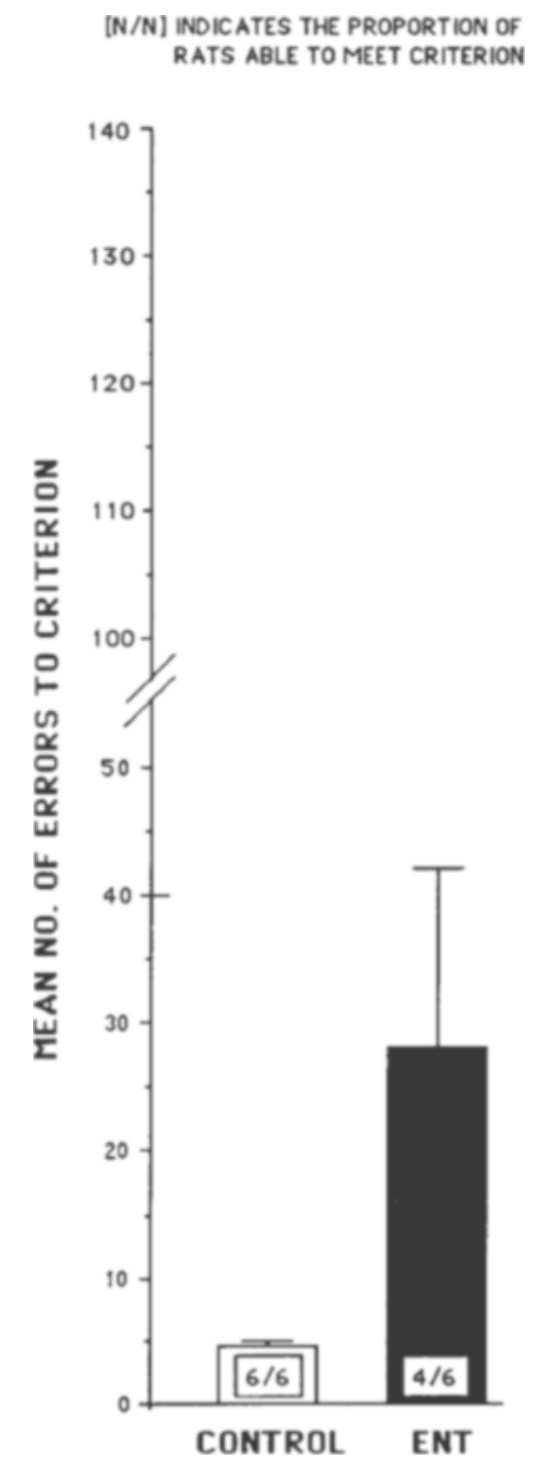

a. DISTRIBUTED TRIALS

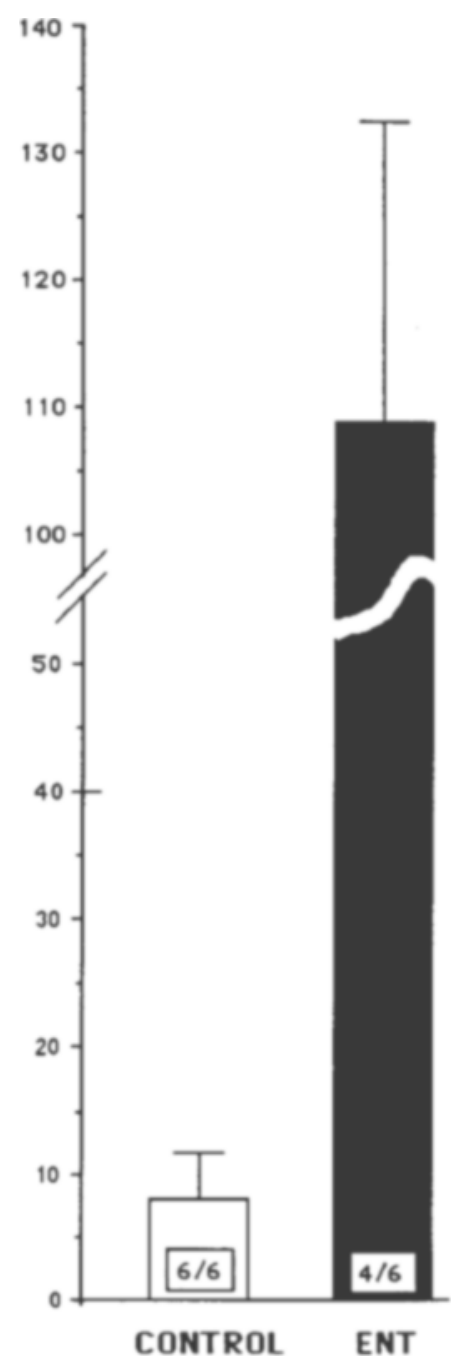

b. MASSED TRIALS

\section{EXPERIMENTAL GROUP}

Figure 5. Mean number of errors to criterion in the spatial reference-memory task. Bars represent the standard error of the mean. ENT = entorhinal-lesioned rats.

Spatial WM. For the distributed-trials groups, all of the controls were able to meet the criterion, whereas only 1 of 6 entorhinal-lesioned rats was able to do so. The entorhinal group required significantly more days to reach the criterion than did the controls $[t(10)=3.111, p=$ $.011]$. During the last 5 days, control rats responded correctly $86 \%$ of the time, whereas entorhinal rats were still performing below chance level with many perseverative errors. In Figure $7 \mathrm{a}$, it can be seen that the entorhinal animals made significantly more errors than did the controls $[t(10)=6.346, p<.001]$.
For the massed-trials groups, the controls were able to meet the criterion by Day 20, whereas none of the lesioned animals was able to do so. During the final 5 days, control rats were able to respond accurately $92 \%$ of the time, whereas entorhinal-lesioned rats continued to perform below chance level due to a strong tendency to perseverate. Figure $7 \mathrm{~b}$ shows that the entorhinal animals made significantly more errors than did the controls $[t(10)$ $=5.227, p<.001]$.

In summary, for both distributed and massed trials, entorhinal-lesioned rats were severely impaired on all 


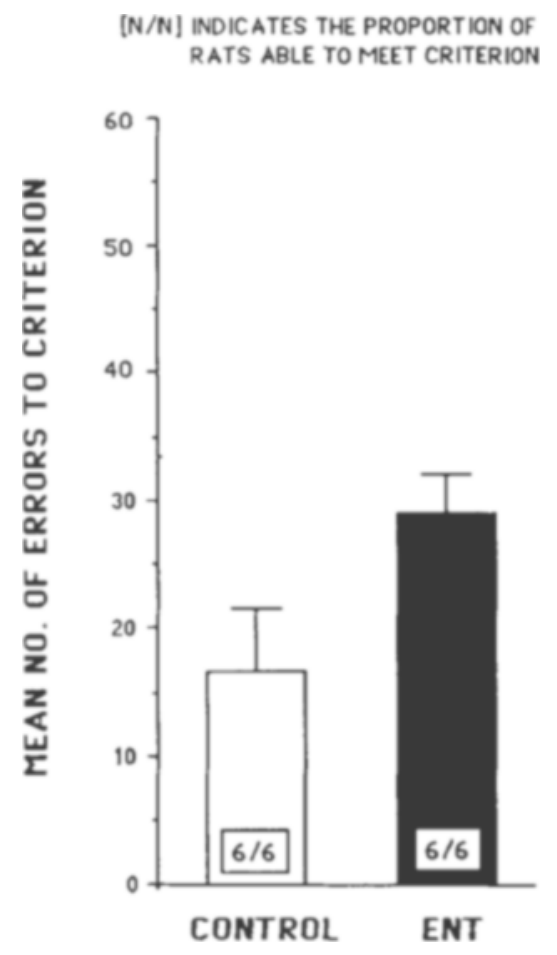

a. DISTRIBUTED TRIALS

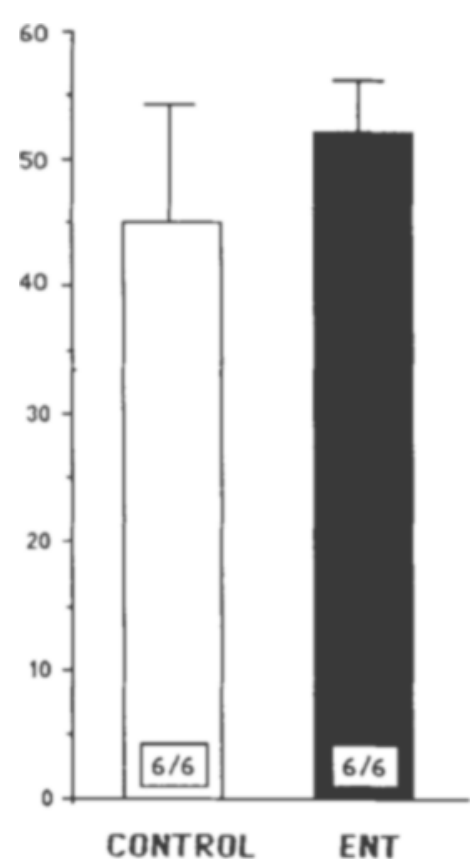

b. MASSED TRIALS

\section{EXPERIMENTAL GROUP}

Figure 6. Mean number of errors to criterion in the nonspatial working-memory task. Bars represent the standard error of the mean. ENT = entorhinal-lesioned rats.

measures of their ability to acquire the spatial WM task, and perseverative responding continued throughout the testing.

Allocentric alternation. This task was relatively easy for both groups of animals. All of the control rats reached criterion for the zero-delay condition within 6 days, and the entorhinal rats that met criterion did so within 10 days. The difference in the mean number of days to criterion was not significant $[t(10)=1.230, p=.247]$. As Figure 8 shows, the difference in the mean number of errors made by the two groups did not differ $[t(10)=1.625$, $p=.135]$. The entorhinal group did make significantly more errors at the 15-, 30-, and 60-sec delay conditions [15-sec delay, $t(10)=2.239, p=.049 ; 30$-sec delay, $t(10)=3.101, p=.011 ; 60$-sec delay, $t(10)=2.588$, $p=.027]$. During the final 3 days of the experiment, control animals were able to choose correctly $91 \%$ of the time, and entorhinal rats $81 \%$ of the time.

In summary, the entorhinal lesions produced impairment (as measured by the number of errors) on this simple allocentric alternation task, except for the zero-delay condition. However, a comparison of the probability levels for the $t$ tests reveals that the lesion effects were less severe than on the other allocentric tasks (spatial RM and WM).

Egocentric alternation. Both the control and the entorhinal groups were able to perform the task, although the ability of individual rats to master it was quite variable. The entorhinal group took significantly fewer days to reach criterion than did the control group $[t(10)=$ $2.330, p=.042$ ]. Figure 9 shows the mean number of errors made by each group for all delay conditions. There was no statistically significant difference between groups at any of the delays [zero delay, $t(10)=1.884, p=.089$; 15-sec delay, $t(10)=0.995, p=.362 ; 30$-sec delay, $t(10)$ $=0.135, p=.896 ; 60$-sec delay, $t(10)=0.032, p=$ $.975]$. During the final 3 days of the experiment, control rats chose correctly $77 \%$ of the time, compared to $76 \%$ for entorhinal-lesioned rats.

In summary, no significant differences in the number of errors to criterion were found between entorhinal and control groups on this difficult egocentric alternation task. There was a trend, however, for the entorhinal rats to perform better than controls at the shortest delay, in that the lesioned animals took significantly fewer days to reach criterion. 


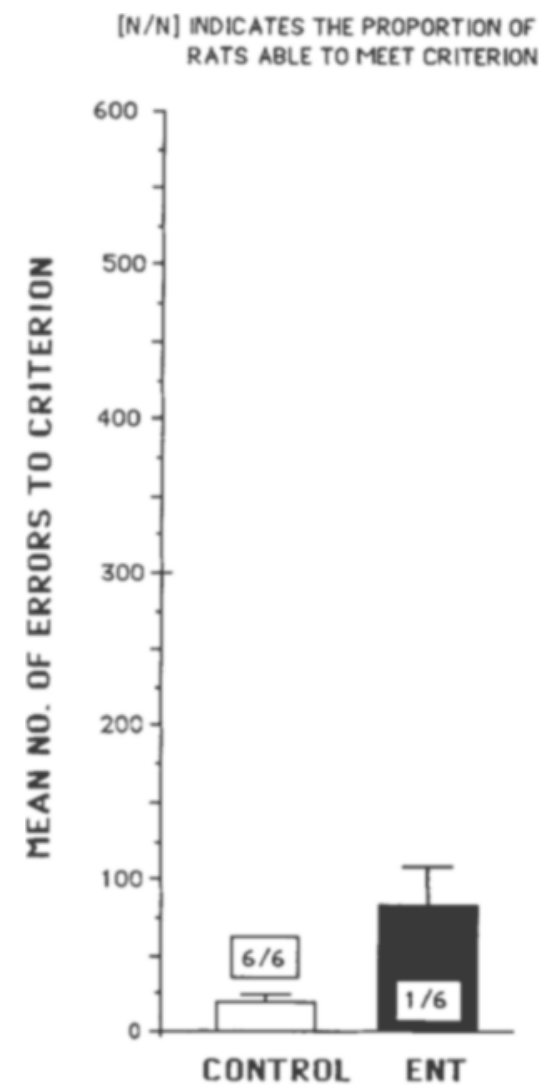

a. DISTRIBUTED TRIALS

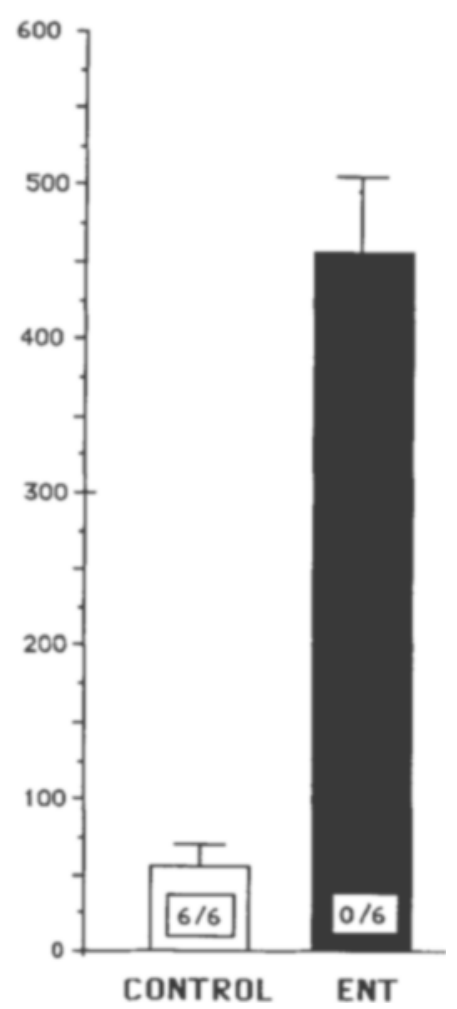

b. MASSED TRIALS

\section{EXPERIMENTAL GROUP}

Figure 7. Mean number of errors to criterion in the spatial working-memory task. Bars represent the standard error of the mean. ENT = entorhinal-lesioned rats.

\section{DISCUSSION}

The present experimental design offered a number of advantages for discrimination among the three theories of hippocampal function under consideration. All of the data were collected using the same apparatus, a constant environment, and consistent procedures. Control of these factors is not typically possible in experiments completed across laboratories.

There were four main lines of evidence from this study that support the conclusion that the entorhinal cortex, and by inference, the hippocampus, are necessary for allocentric spatial responding in the rat. First, the animals with entorhinal lesions were clearly impaired on all of the tasks that required allocentric solution strategies, and the degree of impairment increased disproportionately with task difficulty. Second, animals with entorhinal lesions were generally unimpaired on tasks in which the use of either specific proximal visual-tactile cues or kinesthetic cues was necessary for task solution. Third, the impairment pattern was not a simple function of task difficulty.
Although the spatial and nonspatial RM problems did not differ in difficulty and were identical except for the cues involved in task solution, the entorhinal-lesioned rats were impaired only on the allocentric spatial version. In addition, the greatest impairment was found on the spatial WM problem, yet the lesioned rats were unimpaired on two nonspatial tasks that were more difficult (as defined by the number of trials for control animals to reach asymptotic levels and their proficiency at that level of performance). Finally, although the lesioned rats demonstrated a tendency toward perseverative responding on both the spatial and the nonspatial tasks initially, on the nonspatial problems, this behavior gave way to goal-oriented responding relatively rapidly. On the spatial problems, most lesioned rats continued to perform poorly, and continued to exhibit sporadic perseveration throughout the testing. On the spatial RM task, several lesioned rats were able to solve the problem eventually, whereas on the spatial WM task, none of the entorhinal rats were ever able to solve the task efficiently. These animals continued to engage in a high degree of perseverative responding. This 
[N/N] INDICATES THE PROPORTION OF RATS ABLE TO MEET CRITERION

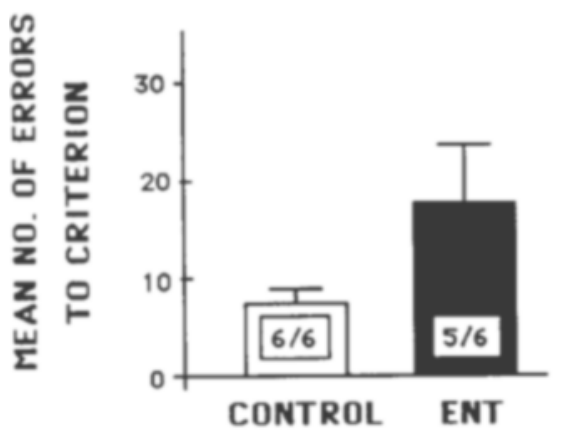

EXPERIMENTAL GROUP ZERO DELAY CONDITION

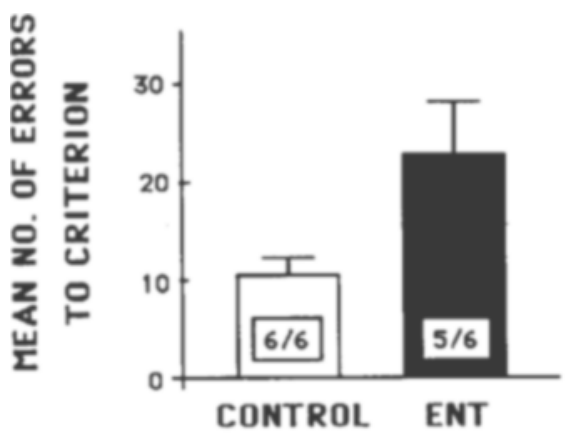

EXPERIMENTAL GROUP 15 SECOND DELAY
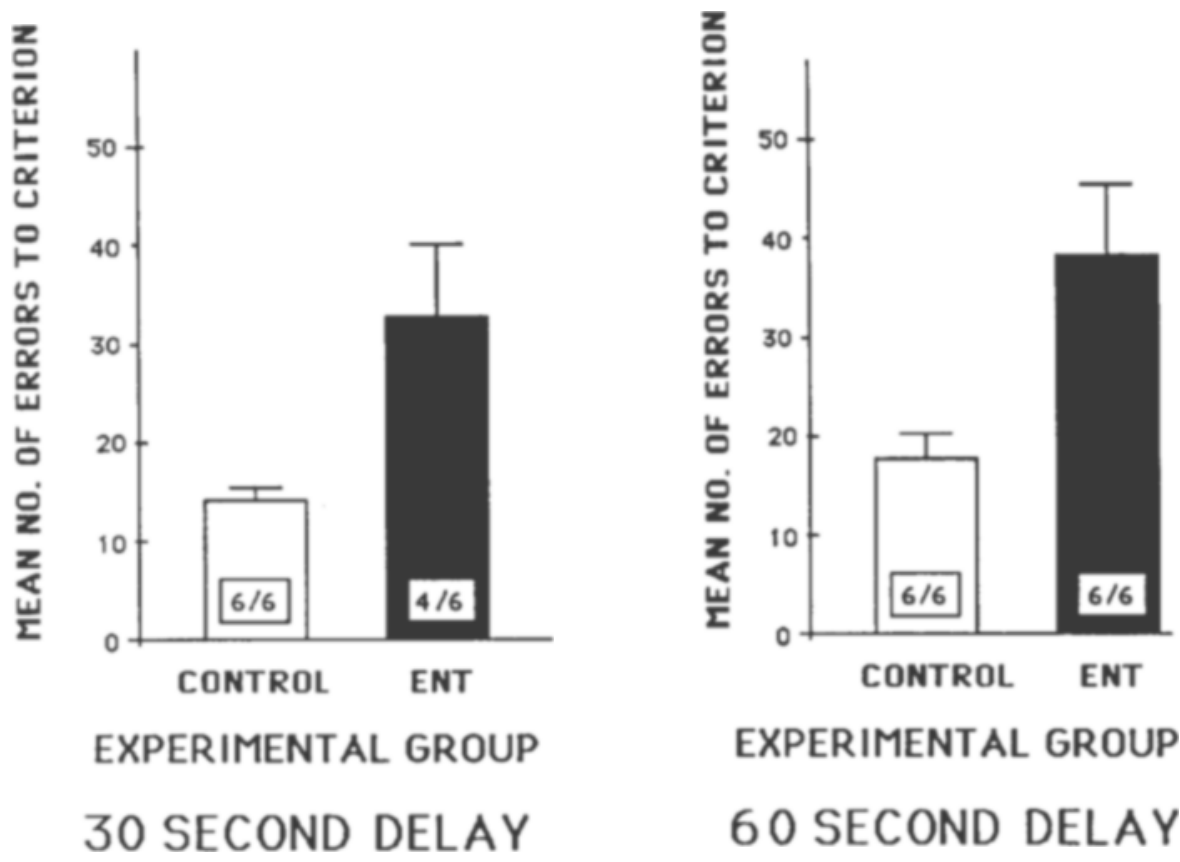

\section{EXPERIMENTAL GROUP}

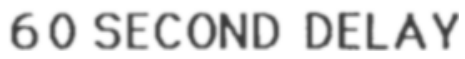

Figure 8. Mean number of errors to criterion in the allocentric alternation task. Bars represent the standard error of the mean. ENT = entorhinal-lesioned rats.

suggests that the representational demands of the spatial WM problem were beyond the capabilities of the damaged animals.

A comparison of distributed and massed trials for control animals revealed an increase, with massed trials, in the number of trials to criterion but a savings in the number of days, on all tasks. Entorhinal-lesioned animals showed the same pattern of savings for the nonspatial problems, but not for the spatial problems. The borderline impairment found for the nonspatial WM problem, in the distributed-trial condition, was most likely a function of initial perseverative responding. If there had been an impairment specific to the representational demands of the task, the massed-trial version should have exacerbated the impairment, but it did not.

The current group of experiments is asymmetrical with respect to the presence or absence of irrelevant, distracting stimuli. However, the outcome pattern was inconsistent with the suggestion that this asymmetry contributed to the impairments found, in any simple way. The lesioned 

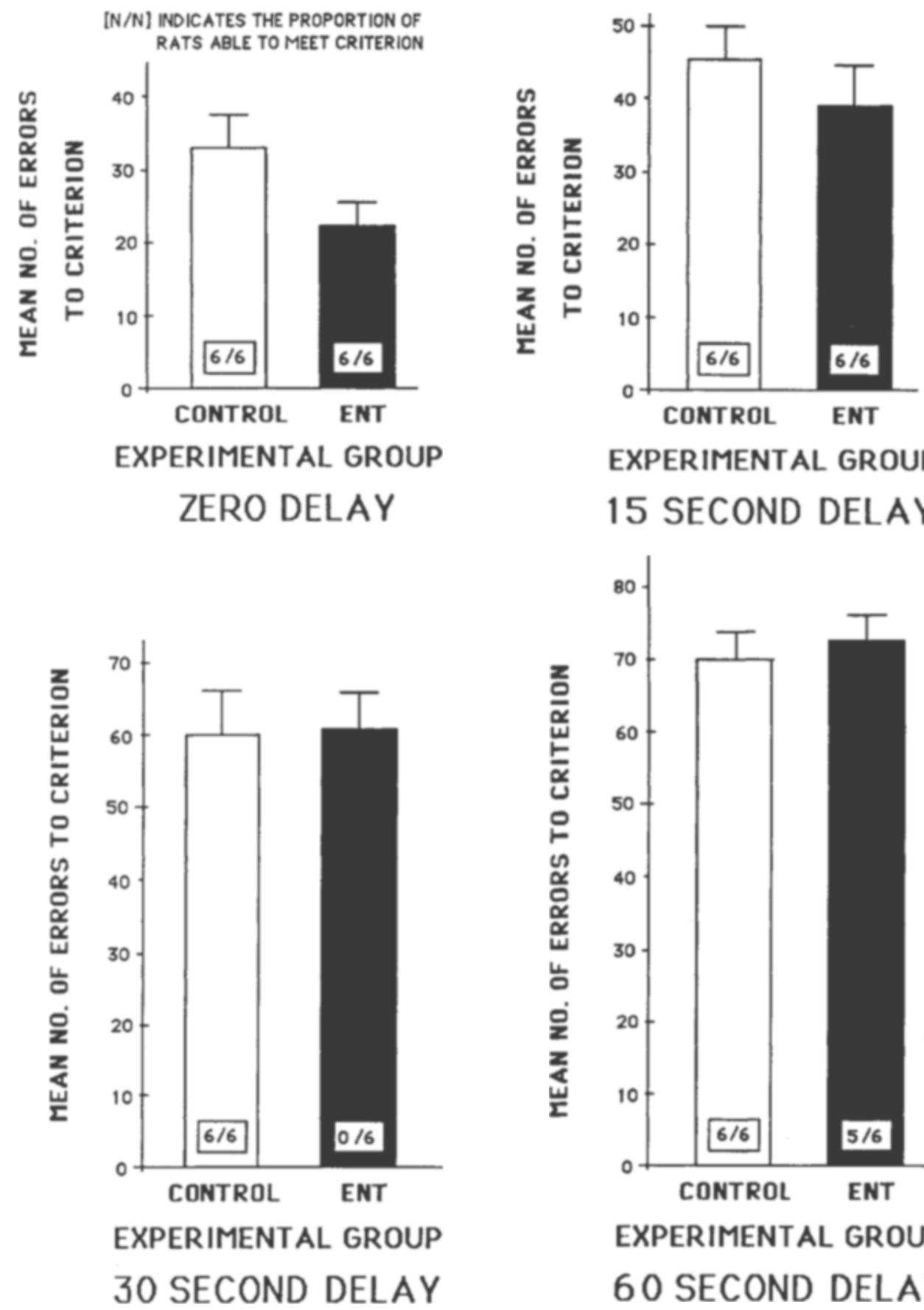

\section{EXPERIMENTAL GROUP \\ 15 SECOND DELAY}

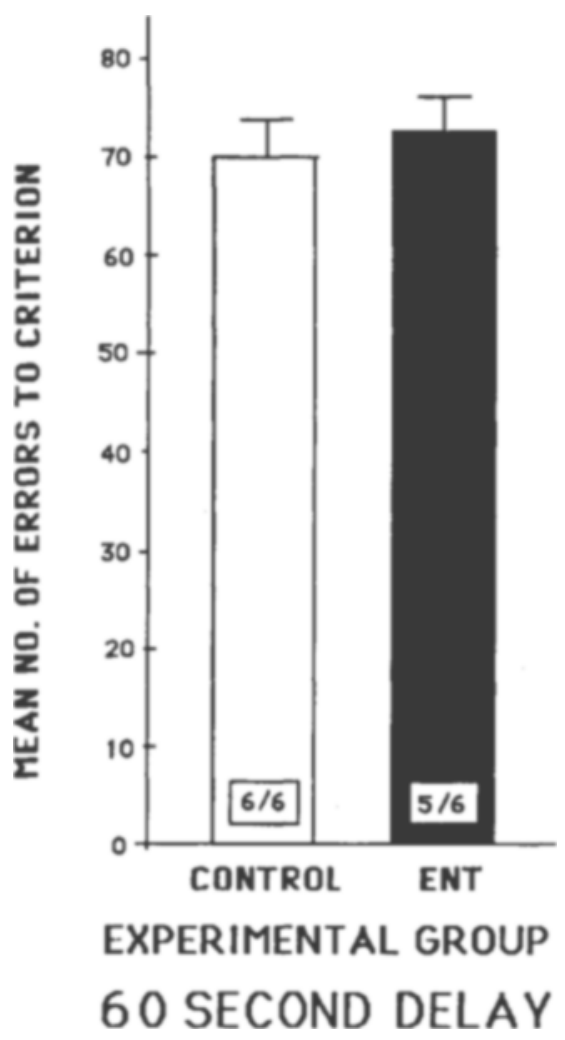

Figure 9. Mean number of errors to criterion in the egocentric alternation task. Bars represent the standard error of the mean. ENT = entorhinal-lesioned rats.

rats in these experiments were impaired on the allocentric tasks, which were not complicated by nonspatial visual-tactile distractors, but they were not impaired on the nonspatial tasks, in which allocentric spatial distractors were present. Restle (1957) demonstrated that the maze behavior of rats is guided by all of the salient, relevant stimuli present but that in an open, elevated maze, place dominance can be expected in a normal rat. Viewed from this perspective, the presence of allocentric distractors may have contributed to the early perseverative behavior of the lesioned rats. The perseveration could have been a function of ineffectual attempts to use a place strategy. Control animals might be expected to discard a place strategy more readily, since they would have a greater capability to recognize that place was not consistently rewarded. If attempts to use an inappropriate place 
strategy were the explanation for response perseveration, it would be expected that the control rats would exhibit some degree of early perseverative behavior on the nonspatial tasks, and this was not observed. It is more likely that response perseveration is a generalized effect of entorhinal lesions.

The data clearly fail to support the WM theory of hippocampal function. Although it is true that the lesioned rats were impaired on both allocentric WM tasks, they were also impaired on the allocentric RM task and unimpaired on the two nonspatial WM tasks (cued and egocentric responding). A comparison of predicted and actual outcomes indicates that three of the six predictions generated from the WM theory were not confirmed by the data.

The predictions generated from TD theory were not supported (again, only three of the six predictions were confirmed). Specifically, the theory predicts that no deficit should occur in spatial RM tasks in hippocampal-damaged rats. However, deficits were found for the entorhinal animals. Furthermore, the theory predicts a nonspatial WM impairment, but this was not observed. Viewed in isolation, there was one finding that could be interpreted as lending support to TD theory. On the allocentric alternation problem, entorhinal-lesioned rats made twice as many errors as did the controls at the zero-delay condition, but this difference did not reach significance. However impairment was found for the 15-, 30-, and 60-sec delay conditions. If the TD theory were correct, a similar pattern of impairment should have been found for the entorhinal group on the egocentric alternation problem. Instead, the entorhinal group made no more errors than did the controls at any of the delay conditions. The above data are also consistent with recent results using a nonspatial WM task in a Y maze (Aggleton, Hunt, \& Rawlins, 1986). In that experiment, 50 different sets of visualtactile, trial-specific cues (a nonmatch-to-sample task) were used, and rats with hippocampal damage were unimpaired relative to controls, even at the 60 -sec interchoice interval. These data are inconsistent with both the TD and WM theories.

The pattern of results from the current experiments are consistent with CM theory (all six predictions are confirmed). These data appear to suggest that the presence of an allocentric component was more crucial than the type of memory system in producing an impairment. However, an examination of the task demands in terms of representational requirements for each type of problem leads to a somewhat different conclusion. Entorhinal rats were vastly more impaired on the spatial WM task than they were on the allocentric alternation and RM tasks. In fact, one third of the entorhinal animals were able to perform the latter two tasks as well as controls by the end of the experiment, whereas another third continued to exhibit sporadic perseveration. Particularly salient cues at or below maze level may have lent to a taxon solution (i.e., an association between movement toward a specific cue and the reinforcer). When there was no salient cue within a few degrees of the goal, it is possible that the lesioned rats were unable to utilize the interrelationship of many distal cues to determine the location of the goal. Thus, the allocentric RM problem may have lent to solutions based on two different representational systems. A similar result was recently noted in a two-odor discrimination RM task using a simultaneous-cue go-left/go-right procedure (Eichenbaum, Fagan, Mathews, \& Cohen, 1988). In that task, rats with hippocampal damage performed as well as controls half of the time. Because the task could be solved using a nonplace strategy, this may have allowed the half of the lesioned rats that chose this strategy to perform as well as controls.

These data suggest that the difficulty with WM theory may reside partly in the fact that although it addresses the distinction between trial-specific stimuli and stimuli that are consistent across trials, it does not address the relationship among stimuli. There is a growing body of evidence that impairment is found on spatial WM tasks because hippocampal animals are unable to process and store the relationships among distal cues in an effective manner. Although entorhinal-lesioned rats in the present study perseverated initially on the nonspatial WM problem, they were eventually able to solve it as well as controls. This may have occurred because there was no intrinsic relationship among the relevant cues.

When Jarrard et al. (1984) tested entorhinal-lesioned rats on spatial and nonspatial RM and WM problems, they found impaired performance for the spatial RM and both the spatial and the nonspatial WM components. One procedural difference-aside from the within-subject design-which may have been important in producing a nonspatial WM deficit, is that the proximal, nonspatial cues were apparently not relocated between each choice. They were rearranged only between trials, according to the procedural description. An intact rat could have used spatial cues in order to avoid revisiting cues, whereas rats with impaired spatial functioning would not have this advantage.

Raffaele and Olton (1988) reported that damage to the fimbria-fornix impairs performance in a delayed nonmatch-to-sample task (a nonspatial WM problem in which the same two visual-tactile cues were used throughout). An analysis of their procedure indicates that in addition to the task-relevant cue, there were at least three other types of cue available for each choice: place, turn direction, and odor. A conditional situation was set up in which the choice could be made by comparing the relative recency of the two stimuli (i.e., if $A$ is more recent, choose $B$, and if $B$ is more recent, choose $A$ ). In addition, during initial training, the rat would have to determine which of the available cues were relevant. The capacity to represent a combination of the various stimuli and their reinforcement contingencies as entities would appear to confer an advantage in this situation. If the hippocampus is necessary for this type of representation, it is not surprising that animals would demonstrate impairment on this preoperatively learned problem. Sutherland, 
Arnold, and Rodriguez (1987) have reported data indicating that the use of recently established spatial representations is disrupted by hippocampal damage.

In addition to being consistent with CM theory, the results of this nonspatial nonmatch-to-sample problem and the data from the current study are compatible with the configural association theory developed by Sutherland and Rudy (1989). This theory can be considered to be an elaboration and extension of Hirsh's $(1974,1980)$ contextual retrieval theory, and of McNaughton's (1987) proposal that the hippocampus contributes to cognitive mapping by storing conditional associations between locations and movements (see also McNaughton \& Nadel, 1989). In Sutherland and Rudy's view, the hippocampus is the neural substrate for the formation of unique neural representations of a combination of stimuli, which can be used to resolve ambiguous situations. Contrasted with this hippocampal-dependent configural association system is a simple association system, which stores separate stimuli among which associations are strengthened or weakened according to the rules of Pavlovian conditioning. Although the results from the present experiments clearly rule out WM and TD theories, they cannot distinguish between $\mathrm{CM}$ and configural assocation theories (the allocentric tests were all configural in nature). Future studies will be required to investigate the points at which these theories diverge.

\section{REFERENCES}

Aggleton, J. P., Hunt, P. R., \& Rawlins, J. N. P. (1986). The effects of hippocampal lesions upon spatial and non-spatial tests of working memory. Behavioural Brain Research, 19, 133-146.

BARNEs, C. A. (1988). Spatial learning and memory processes: The search for their neurobiological mechanisms in the rat. Trends in Neuroscience, 11, 163-169.

Eichenbaum, H., Fagan, A., Mathews, P., \& Cohen, N. J. (1988). Hippocampal system dysfunction and odor discrimination learning in rats: Impairment or facilitation depending on representational demands. Behavioral Neuroscience, 102, 331-339.

HiRsH, R. (1974). The hippocampus and contextual retrieval of information from memory: A theory. Behavioral Biology, 12, 421-444.

HIRSH, R. (1980). The hippocampus, conditional operations, and cognition. Physiological Psychology, 8, 175-182.

HoNIG, W. K. (1978). Studies of working memory in the pigeon. In S. H. Hulse, H. Fowler, \& W. K. Honig (Eds.), Cognitive processes in animal behavior (pp. 211-248). Hillsdale, NJ: Erlbaum.

JARRARD, L. E. (1980). Selective hippocampal lesions and behavior. Physiological Psychology, 8, 198-206.

JARRARD, L. E. (1983). Selective hippocampal lesions and behavior: Effects of kainic acid lesions on performance of place and cue tasks. Behavioral Neuroscience, 97, 873-889.

JarRard, L. E., OKaichi, H., Steward, O., \& Goldschmidt, R. B. (1984). On the role of hippocampal connections in the performance of place and cue tasks: Comparisons with damage to hippocampus. Behavioral Neuroscience, 98, 946-954.

MARR, D. (1971). Simple memory: A theory for archicortex. Philosophical Transactions of the Royal Society of London, B262, 23-82.
MCNaughton, B. L. (1987). Neural association of movement and space: Preliminary steps toward a noncartographic theory of spatial representation and learning. Neuroscience Letters (Suppl. 29), S143-S144.

MCNaughton, B. L., \& NADEL, L. (1989). Hebb-Marr networks and the neurobiological representation of action in space. In $\mathbf{M}$. A. Gluck \& D. E. Rumelhart (Eds.), Neuroscience and connectionist theory (pp. 1-63). Hillsdale, NJ: Erlbaum.

MoRris, R. G. M. (1983). An attempt to dissociate "spatial-mapping" and "working-memory" theories of hippocampal function. In W. Seifert (Ed.), Neurobiology of the hippocampus (pp. 405-430). London: Academic Press.

Morris, R. G. M., Garrud, P., Rawlins, J. N. P., \& O'Keefe, J. (1982). Place navigation impaired in rats with hippocampal lesions. Nature, 297, 681-683.

NIKI, H. (1966). Response perseveration following the hippocampal ablation in the rat. Japanese Psychological Research, 8, 1-9.

O'KeEFE, J., NADEL, L. (1978). The hippocampus as a cognitive map. Oxford: Clarendon Press.

O'KeEFE, J., \& NADEL, L. (1979). Precis of O'Keefe \& Nadel's “'The hippocampus as a cognitive map." Behavioral \& Brain Sciences, 2 , 487-533.

O'Keefe, J., Nadel, L., Keightley, S., \& Kill, D. (1975). Fornix lesions selectively abolish place learning in the rat. Experimental Neurology, 48, 152-166.

OLton, D. S. (1983). Memory functions and the hippocampus. In W. Seifert (Ed.), Neurobiology of the hippocampus (pp. 335-373). London: Academic Press.

Olton, D. S., Becker, J. T., \& Handelmann, G. E. (1979). Hippocampus, space and memory. Behavioral \& Brain Sciences, 2, 313-322.

Olton, D. S., \& FeustLe, W. (1981). Hippocampal function and nonspatial memory. Experimental Brain Research, 41, 380-389.

Olton, D. S., \& PAPAs, B. C. (1979). Spatial memory and hippocampal function. Neuropsychologia, 17, 669-682.

Olton, D. S., Walker, J. A., \& Wolf, W. A. (1982). A disconnection analysis of hippocampal function. Brain Research, 233, 241-253.

PAXINos, G., \& WATSON, C. (1982). The rat brain in stereotaxic coordinates. Sydney: Academic Press.

Racine, R. J., \& Kimble, D. P. (1965). Hippocampal lesions and delayed alternation in the rat. Psychonomic Science, 3, 285-286.

Raffaele, K. C., \& Olton, D. S. (1988). Hippocampal and amygdaloid involvement in working memory for nonspatial stimuli. $B e$ havioral Neuroscience, 102, 349-355.

Rawlins, J. N. R. (1985). Associations across time: The hippocampus as a temporary memory store. Behavioral \& Brain Sciences, 8 , 479-496.

Rawlins, J. N. R., OLton, D. S. (1982). The septo-hippocampal system and cognitive mapping. Behavioural Brain Research, 5, 331-358.

RESTLE, F. (1957). Discrimination of cues in mazes: A resolution of the "place-vs.-response" question. Psychological Review, 64, 217-228.

Sutherland, R. J., ARnold, K. A., \& Rodriguez, A. R. (1987). Anterograde and retrograde effects on place memory after limbic or diencephalic damage. Society for Neuroscience Abstracts, 13, 1066.

Sutherland, R. J., \& Rudy, J. W. (1989). Configural association theory: The role of the hippocampal formation in learning, memory, and amnesia. Psychobiology, 17, 129-144.

Tномаs, G. J. (1978). Delayed alternation in rats after pre- or postcommissural fornicotomy. Journal of Comparative \& Physiological Psychology, 92, 1128-1136.

(Manuscript received April 17, 1989; revision accepted for publication July 20, 1989.) 